\title{
Non-invasive cell tracking of SPIO labeled cells in an intrinsic regenerative environment: The axolotl limb
}

\author{
HENRIK LAURIDSEN $^{1}$, CASPER BINDZUS FOLDAGER ${ }^{2}$, LINE HANSEN ${ }^{3}$ and MICHAEL PEDERSEN ${ }^{1}$ \\ ${ }^{1}$ Department of Clinical Medicine, Comparative Medicine Lab, Aarhus University, 8200 Aarhus N; \\ ${ }^{2}$ Department of Clinical Medicine, Orthopaedic Research Lab, Aarhus University; ${ }^{3}$ Interdisciplinary \\ Nanoscience Center (iNANO), Aarhus University, 8000 Aarhus C, Denmark
}

Received August 4, 2017; Accepted October 27, 2017

DOI: $10.3892 /$ etm.2018.5865

\begin{abstract}
Non-invasive methods to track the progress of stem cell therapies are important in the development of future regenerative therapies. Super-paramagnetic iron oxide particles (SPIOs) have previously been applied to track cells using magnetic resonance imaging (MRI) in vivo in non-regenerative animal models. To the best of the author's knowledge, the present study investigated for the first time, the feasibility of tracking SPIO labeled cells in an intrinsic regenerative environment, the regenerating limb of the axolotl, and investigated the homing of stem cell-like blastema cells to the regenerative zone. Viability and labeling success of labeled axolotl blastema cells was tested in vitro using cell culture and histology. SPIO labeling was performed in situ by intramuscular injections and mapped using MRI. Enhanced permeability and retention (EPR) effects were evaluated in the blastema, liver, heart, kidney and a back muscle. Finally, SPIO/Fluorophore-labeled blastema cells were injected intravascularly and tracked using MRI and fluorescence imaging. It was demonstrated that SPIO labeling had no effect on axolotl cell viability in vitro. In situ labeling resulted in an MRI signal alteration during 48 days of regeneration. EPR effect of unbound SPIO was observed only in the liver. MRI tracking revealed increased concentrations
\end{abstract}

Correspondence to: Dr Henrik Lauridsen, Department of Clinical Medicine, Comparative Medicine Lab, Aarhus University, Palle Juul-Jensens Boulevard 99, 8200 Aarhus N, Denmark

E-mail: henrik@clin.au.dk

Abbreviations: DLS, dynamic light scattering; dpa, days post amputation; EPR, enhanced permeability and retention; GFP, green fluorescent protein; IVIS, in vivo imaging system; KL, Kullback-Leibler divergence; L, length; MRI, magnetic resonance imaging; PEG, polyethylene glycol; PLL, poly-l-lysine; ROI, region-of-interest; Si-PEG, 2-methoxy(polyethyleneoxy)-propyltrimethoxysilane; SKL, symmetric Kullback-Leibler divergence; SPIO, super-paramagnetic iron oxide particle; TE, echo time; TEA, triethylamine; TF6, Tide Fluor 6; THTA, tris-[1-(3-hydroxypropyl)triazol-4-ylmethyl]amine; $\mathrm{TR}$, repetition time; $\mathrm{W}$, weight

Key words: ferumoxides, MRI, cell tracking, axolotl, stem cells, regenerative medicine of SPIO labeled blastema cells in the liver, kidney and heart, however not the blastema of intravascularly injected axolotls. In conclusion, the results demonstrated that SPIO labeling facilitated non-invasive tracking of injected cells in the regenerating axolotl limb. An early homing mechanism of injected blastema cells to an injury site was not observed.

\section{Introduction}

Tissue regeneration is a regulative process widespread in most classes of animals that maintains or reestablishes the normal functionality of cells, tissues, and in some spectacular cases even major parts of organs and appendages (1-4). Unfortunately, our own mammalian class holds very limited regenerative potential and relies heavily on fibrosis and scar formation following injury (3,5-10). Before regenerative therapies can become a reality, however, our understanding of underlying mechanisms needs to be improved and effective techniques for monitoring the regenerative progress have to be developed. One way of investigating regenerative phenomena is by applying the August Krogh Principle: 'For a large number of problems there will be some animal of choice or a few such animals on which it can be most conveniently studied' (11). In fact, there are some vertebrate species for which this statement in a regenerative perspective applies very well: The urodele amphibians. Urodele amphibians, salamanders and newts generally possess a very high degree of regenerative capacity (12-16). Cardiac tissue, intestines, liver, skeletal muscle, central and peripheral nervous system, lens, retina, jaw, and even whole appendages such as limbs and tail are examples of regenerative structures of these animals (17). Particular attention has been drawn to the endemic Mexican salamander, the axolotl [Ambystoma mexicanum (Shaw and Nodder, 1798)], due to its impressive regenerative potential and easy maintenance $(18,19)$. Axolotl limb regeneration has been extensively studied $(3,13,17,20-23)$ and is characterized by a three-step regenerative process: Wound healing, blastema formation and regrowth $(3,17,23)$. Within the first couple of hours following amputation of a limb the wound is sealed with a wound epidermis by migrating cells from the adjacent epidermis. Within 1-2 weeks, neurotrophic and epidermal regulation induces dedifferentiation of differentiated cells adjacent to the amputation site leading to the formation of a 
structure termed a blastema containing dedifferentiated cells with varying origin (e.g., connective tissue, muscular tissue, bone and nerves). Finally, dedifferentiated stem cell-like blastema cells proliferate, redifferentiate and restore the missing limb. In the present study we chose the axolotl as animal model in order to monitor a complete intrinsic regenerative process.

Before regenerative therapies can ever come to play, an appropriate monitoring technology has to be developed that ensures non-invasive follow up examinations of patients undergoing therapy (24-26). In studies evaluating the effectiveness of stem cell-based regenerative therapies, monitoring has traditionally relied on histological techniques. In order to detect the presence of cells within the region of interest, test animals are usually sacrificed, and biopsies are collected and evaluated using histology $(27,28)$. Even though these methods and techniques are valuable in a research setting, they preclude non-invasive in vivo assessment and longitudinal tracking of therapeutic progress.

Stem or progenitor cell fate can be monitored by an alternative method by labeling cells of interest with non-toxic super-paramagnetic iron oxide particles (SPIOs) that allow for in vivo cell tracking using magnetic resonance imaging (MRI) (24). Since SPIOs are non-toxic to the labeled cells, this methodology is minimally invasive and completely safe due to the harmless nature of MRI (29). SPIOs are either internalized by the endosomal-lysosomal pathway or bind to the surface of cells, and due to their magnetic properties, they increase the magnetic susceptibility and decrease the MRI-measured properties of water, especially the spin-spin (T2) and to some degree the spin-lattice (T1) relaxation times (30). SPIO labeling has successfully been used to track stem cell migration and quantify the number of cells arriving in the target zone $(31,32)$. At present the SPIO labeling technique has been applied in a number of preclinical studies, but to our knowledge never in a system with true intrinsic regenerative capacity.

The purpose of the present study was to introduce SPIO labeling for cell tracking in a truly regenerative environment, the regenerating limb of the axolotl. This method was subsequently used to investigate an early homing effect of blastema cells to a regenerative zone when applied intravascularly.

\section{Materials and methods}

Animals, husbandry and ethics. The procedures in this study were carried out in accordance to the National and Institutional Legislation for Care and Use of Laboratory animals. The experimental protocol was approved by the Danish Animal Experiments Inspectorate (protocol no. 2012-15-2934-00353). Animals used in this study were Mexican axolotls (Ambystoma mexicanum) obtained from a commercial breeder (Exoterra GmbH, Holzheim, Germany). Animals were housed individually in plastic containers with a $10 \mathrm{~cm}$ water depth and a $930 \mathrm{~cm}^{2}$ surface area with regular water change and a 12-h light:dark cycle. They were fed every second day with protein-enriched trout pellets. Anesthesia was obtained using $200 \mathrm{mg} / \mathrm{l}$ ethyl-4-aminobenzoate.

Nanoparticles. To increase the broadness of the experiments, two commercially available and one custom designed SPIO, all with similar MRI properties, were applied. First, the SPIO,
Resovist (Bayer Schering Pharma AG, Berlin, Germany), was applied for viability testing and enhanced permeability and retention experiments. This relatively large polycrystalline, polydisperse carboxydextran-coated particle (core diameter: $4.2 \mathrm{~nm}$, hydrodynamic diameter: $45-60 \mathrm{~nm}$ ), provides a high MRI relaxation enhancement ( $\mathrm{R} 1$ relaxivity: $7.2 \mathrm{mM}^{-1} \mathrm{sec}^{-1}$; $\mathrm{R} 2$ relaxivity: $82 \mathrm{mM}^{-1} \mathrm{sec}^{-1}$; at $1.5 \mathrm{~T}$ and $310 \mathrm{~K}$ ), and has been applied for detection and characterization of small focal liver lesion and immune cell and stem cell labeling (33). Second, an ultra-small SPIO, VSOP C-200 (Ferropharm GmbH, Teltow, Germany), was applied for viability testing and in situ labeling. This small citrate coated particle (core diameter: $4 \mathrm{~nm}$, hydrodynamic diameter: $7 \mathrm{~nm}$ ) effects $\mathrm{T} 1$ and $\mathrm{T} 2$ relaxation (R1 relaxivity: $13.97 \mathrm{mM}^{-1} \mathrm{sec}^{-1}$; $\mathrm{R} 2$ relaxivity: $33.45 \mathrm{mM}^{-1} \mathrm{sec}^{-1}$; at $1.0 \mathrm{~T}$ and $310 \mathrm{~K}$ ), and has been applied for tumor imaging and cell labeling $(34,35)$. Third, a custom made SPIO was synthesized as described earlier (36) and applied for enhanced permeability and retention experiments and testing of the homing capabilities of blastema cells. This particle effects T2 relaxation (R2 relaxivity: $119 \mathrm{mM}^{-1} \mathrm{sec}^{-1}$; at $1.5 \mathrm{~T}$ and $293 \mathrm{~K}$, and $92 \mathrm{mM}^{-1} \mathrm{sec}^{-1}$; at $3.0 \mathrm{~T}$ and $293 \mathrm{~K}$ ). For the enhanced permeability and retention experiment the custom made SPIO particles were coated with polyethylene glycol (PEG). A total of $5 \mathrm{mg}$ oleic acid coated particles were dissolved in $2 \mathrm{ml}$ toluene in a glass tube and $10 \mu \mathrm{l} 2$-methoxy(polyethyleneoxy)-propyltrimethoxysilane (Si-PEG), Mw=460-590 (Gelest Inc., Morrisville, PA, USA) were added together with $50 \mu \mathrm{l}$ triethylamine (TEA) and $50 \mu \mathrm{l} \mathrm{H}_{2} \mathrm{O}$ while stirring $(161 \mathrm{x} \mathrm{g})$. The mixture was incubated at room temperature for $24 \mathrm{~h}$. The solution was heated to $105^{\circ} \mathrm{C}$ for $30 \mathrm{~min}$ and the particles were precipitated with pentane and the supernatant was discarded. The particles were then re-dissolved in toluene and precipitated with pentane. This washing procedure was repeated three times, and the particles were resuspended in water. The sample was centrifuged at 11,180 x g for $2 \mathrm{~min}$ three times to remove any aggregates. The hydrodynamic size of the pegylated particles was measured using dynamic light scattering (DLS) and revealed a hydrodynamic size of $23 \mathrm{~nm}$ (number mean of three measurements) hence we named this custom made particle SPIO-PEG (23 nm).

For the in situ cell labeling experiment, the same custom made SPIO particle was coated with PEG and conjugated to a Tide Fluor 6 (TF6) alkyne fluorophore from AAT Bioquest allowing for dual modality imaging (MRI and optical imaging). A total of $20 \mathrm{mg}$ oleic acid coated particles were dissolved in $2 \mathrm{ml}$ toluene in a glass tube. A total of $60 \mathrm{mg}$ Azido-PEG-Si(OMe) ${ }_{3}\left(\mathrm{Si}-\mathrm{PEG}-\mathrm{N}_{3}\right)$, Mw 3,000 kDa (Iris-Biotech) was dissolved in $2 \mathrm{ml}$ toluene and added to the particle solution together with $100 \mu \mathrm{l}$ TEA and $100 \mu \mathrm{l} \mathrm{H}_{2} \mathrm{O}$ during stirring $(161 \mathrm{x} \mathrm{g})$. The mixture was incubated at room temperature for $24 \mathrm{~h}$ and washed as described above. The Tide Fluor ${ }^{\mathrm{TM}} 6$ alkyne was conjugated to the azide-particles by a copper(I)-catalyzed Huisgen cycloaddition reaction. A click stem solution was made from $6 \mathrm{mM} \mathrm{CuSO}_{4}, 200 \mathrm{mM}$ sodium-ascorbate, $50 \mathrm{mM}$ tris-[1-(3-hydroxypropyl)-triazol4-ylmethyl]amine ligand (THTA) and $\mathrm{H}_{2} \mathrm{O}$. The click stem solution was mixed with $200 \mu \mathrm{g}$ azide-particles and $0.012 \mathrm{mg}$ Tide Fluor ${ }^{\mathrm{TM}} 6$ alkyne. The click stem solution made up a fourth of the total volume of this reaction solution. After $2 \mathrm{~h}$ at room temperature and $28 \mathrm{xg}$, the particles were put into 
dialysis bags (Spectra/POR ${ }^{\mathrm{R}}$ Dialysis membrane, $\mathrm{MWCO}$ 12-14000; Spectrum Laboratories, Inc., Rancho Dominguez, CA, USA) and dialysed against PBS with 10 shifts. The hydrodynamic size measured with DLS was $47 \mathrm{~nm}$ (number mean of three measurements); hence we named this particle SPIO-PEG-TF6 (47 nm).

Viability and SPIO labeling of cultured blastema cells. Early bud blastema cells were harvested from 7 large axolotls [Weight (W), $95.75 \pm 15.5 \mathrm{~g}$; Length (L), $22.7 \pm 1.0 \mathrm{~cm}$ ], dissociated as described earlier (37), distributed into 55 wells (96-well plates) and incubated in growth medium (50\% Leibovitz's L-15, 15\% fetal bovine serum, and 35\% phosphate-buffered saline) with either $0 \mathrm{mg} \mathrm{Fe} / \mathrm{L}$ [C1 (n=11), C2 (n=11)] or $50 \mathrm{mg} \mathrm{Fe} / \mathrm{L}$ from VSOP ( $\mathrm{n}=11)$, Resovist $(\mathrm{n}=11)$ or Resovist and the transfection agent poly-l-lysine (PLL) $(\mathrm{n}=11)$ for $24 \mathrm{~h}$ at room temperature and atmospheric air. After incubation with SPIO, cells were washed and transferred to growth medium. Blastema cell viability was investigated using a PicoGreen assay (Invitrogen; Thermo Fisher Scientific, Inc., Waltham, MA, USA) after 1 week (C1) and 3 weeks (C2, VSOP, Resovist, Resovist/PLL) of culture.

In situ labeling. Twelve small axolotls ( $\mathrm{W}, 7.5 \pm 2.1 \mathrm{~g}$; $\mathrm{L}, 10.2 \pm 1.1 \mathrm{~cm}$ ) were anaesthetized, and a $50 \mu 1$ bolus of either $50 \mathrm{mg} \mathrm{Fe} / \mathrm{L}$ VSOP $(\mathrm{n}=6)$ or saline $(\mathrm{n}=6)$ was injected intramuscularly in the muscles lining the femur. To increase labeling success, animals were kept under light anesthesia ( $20 \mathrm{mg} / \mathrm{l}$ ethyl p-aminobenzoate) and cooled to $5^{\circ} \mathrm{C}$ overnight. Animals were subjected to MRI (1.5 T Siemens Magnetom Avanto; gradient-echo sequence; TR, $10.7 \mathrm{msec}$; TE, $4.42 \mathrm{msec}$; $\theta, 25^{\circ}$; spatial resolution, $0.3 \times 0.3 \times 0.4 \mathrm{~mm}^{3}$; FOV depending on sample size), and imaging was performed at 0 (pre amputation), and 2, 9, 16, 28, 49, 84 days post amputation (dpa). The regenerating blastema appeared at $16 \mathrm{dpa}$.

Presence of SPIOs resulted in decreased signal intensity in labeled areas easily recognizable by visual inspection of acquired MR images. To objectively compare pixel distributions the Kullback-Leibler divergence test was applied (38). A region-of-interest (ROI) within the limb stump and a ROI within the protruding blastema (only after $16 \mathrm{dpa}$ ) were selected for comparison with similar sized regions within the contralateral unlabeled limb. Signal intensities were converted to 8-bit grayscale and histograms were generated displaying normalized pixel distributions. The Kullback-Leibler divergence can be regarded as a dissimilarity measure between two arbitrary probability distributions (39). Given the two distributions $P$ and $Q$ the Kullback-Leibler divergence (KL) is defined as:

$$
K L(P, Q)=\int_{-\infty}^{\infty} p(x) \log \frac{p(x)}{q(x)} d x
$$

Always non-negative the Kullback-Leibler divergence is 0 if and only if $P=Q$. As a result of this, as the signal intensity in the labeled limb theoretically approaches that of the contralateral unlabeled limb over time due to SPIO dilution, the Kullback-Leibler divergence will approach 0. Between each histogram pair (P and Q) the symmetric Kullback-Leibler divergence (SKL) defined as $\operatorname{SKL}(P, Q)=1 / 2 \mathrm{KL}(P, Q)+1 / 2$ $\mathrm{KL}(Q, P)$ was calculated. As $\log _{10}(\mathrm{SKL})$ can be approximately treated as normally distributed, a one-way ANOVA was applied to the $\log _{10}$ (SKL) of each MRI data collection point.

The fully regenerated blastema ( $84 \mathrm{dpa}$ ) was sectioned and histologically examined with H\&E and Prussian blue staining to investigate the presence of any remaining SPIOs.

Enhanced permeability and retention. Amputations were performed on 9 small animals $(\mathrm{W}, 8.1 \pm 0.7 \mathrm{~g} ; \mathrm{L}, 11.2 \pm 0.6 \mathrm{~cm}$ ) to evaluate the enhanced permeability and retention (EPR) effect during axolotl regeneration. At early bud stage (13 dpa), animals underwent MRI and T2 maps were produced (1.5 T Siemens Magnetom Avanto; Multiple spin-echo sequence; TR, $14,720 \mathrm{msec}$; TE, 39, 207 and $337 \mathrm{msec} ; \theta, 150^{\circ}$; spatial resolution, $0.625 \times 0.625 \times 0.18 \mathrm{~mm}^{3}$; FOV depending on sample size). Subsequently, intracardial injections were performed with $50 \mu \mathrm{l}(\sim 12.5 \%$ of blood volume) of either $5 \mathrm{mg} \mathrm{Fe} /(\mathrm{kg}$ body weight) Resovist ( $\mathrm{n}=3$ ) or $5 \mathrm{mg} \mathrm{Fe} /(\mathrm{kg}$ body weight) SPIO-PEG $(23 \mathrm{~nm})(\mathrm{n}=6)$. MRI was repeated $24 \mathrm{~h}$ after injection of contrast agent at $14 \mathrm{dpa}$. T2 was measured in ROIs placed in the blastema, liver, heart, a back muscle, and kidney.

Homing of blastema cells. Amputations were performed on 12 large donor animals $(\mathrm{W}, 48.3 \pm 5.7 \mathrm{~g} ; \mathrm{L}, 19.7 \pm 1.1 \mathrm{~cm})$ and 12 small receiver animals $(\mathrm{W}, 7.8 \pm 0.9 \mathrm{~g} ; \mathrm{L}, 11.2 \pm 0.5 \mathrm{~cm}$ ) to investigate whether blastema cells possess the ability to home at the regenerative zone. At early bud stage, cells were harvested from donor animal blastemas and dissociated as described above, separated into two vials and incubated for $24 \mathrm{~h}$ with $50 \mathrm{mg} \mathrm{Fe} / \mathrm{L}$ SPIO-PEG-TF6 (47 nm) or without SPIO respectively. Labeling success was evaluated with Prussian blue staining of histologically sectioned coagel single cell suspensions of labeled and unlabeled cells. SPIO-PEG-TF6 (47 $\mathrm{nm})$ labeled and unlabeled cells were administered to the receiver animals through intracardial injections of 120,000 unlabeled cells pr. control animal $(n=6)$ and 120,000 SPIO labeled cells pr. SPIO treated animal $(n=6)$. Twenty four $\mathrm{h}$ after injections, animals underwent MRI (3.0 T Siemens Magnetom Skyra; Multiple spin-echo sequence; TR, $6.130 \mathrm{msec}$; TE, 25, 50, 75, 100 125, 150, 175, 200, 225, 250, $275,300,325,350,375$ and $400 \mathrm{msec} ; \theta, 180^{\circ}$; spatial resolution, $0.417 \times 0.417 \times 0.8 \mathrm{~mm}^{3}$; FOV depending on sample size). Additionally, animals were optically scanned using an in vivo imaging system (IVIS Spectrum pre-clinical in vivo imaging system, PerkinElmer). A spectral unmixing epi-illumination protocol with multiple emission and excitation wavelengths was applied (Em/Ex: 780/675, 760/675, 740/675, 720/675, 720/640, 700/640, 780/605, 760/605, 740/605, 720/605, 700/605, 680/605, 660/605 nm).

An additional non-MRI cell tracking experiment designed to test the potential up-concentration of injected blastema cells on a gross anatomical level was carried out in 10 large axolotls (W, $53.8 \pm 13.9 \mathrm{~g} ; 1,19.2 \pm 1.9 \mathrm{~cm}$ ). Amputation was induced in three globally green fluorescent protein (GFP) expressing donor axolotls $\left(\mathrm{GFP}^{+}\right)$, two non-GFP (GFP-) donor animals, and five GFP' receiver animals. Blastema cells were harvested and dissociated as described above at the early bud stage and administered to the five GFP- receiver animals through intracardial injections of 120,000 cells each $\left(\mathrm{GFP}^{+}\right.$: $n=3$, GFP $: n=2$ ). After $24 \mathrm{~h}$, receiver animals were sacrificed, and the blastema, liver, back muscle, heart, kidney, and lungs 
were resected and inspected for presence of $\mathrm{GFP}^{+}$cells with microscopy.

To make sure that an early bud blastema is adequately supplied by blood to allow for vascularly injected blastema cells to reach the blastema, ultrasound examination of 5 animals (W, $47.6 \pm 6.8 \mathrm{~g} ; \mathrm{L}, 18.8 \pm 0.9 \mathrm{~cm}$ ) was carried out as the early bud blastema stage was reached using a 40-50 MHz ultrasound system (VisualSonics Vevo 2100; Transducer: MS700; Fujifilm VisualSonics, Inc., Toronto, ON, Canada). Color Doppler imaging was applied to visualize overall blood flow and quadratic averaging was performed on frames acquired in B-mode to visulaze small vessels supplying the regenerating limb. Blood flow in the main artery supplying the blastema was measured using pulsed wave Doppler ultrasound.

Statistical analysis. Data were analyzed using statistical software Stata 12 (StataCorp LP, College Station, Texas, USA) using Students t-test and one-way ANOVA when appropriate, significance level $\alpha<0.05$. Bonferroni correction was applied for multiple comparisons. A custom made Matlab (Matlab R2012b; Mathworks, Natick, MA, USA) function was applied for Kullback-Leibler analysis.

\section{Results}

Viability of SPIO labeled blastema cells. Blastema cells do not tolerate culturing outside their natural milieu for extended periods very well (18). A significant decline in cell abundance was observed in all cultures from one to three weeks of culturing (Fig. 1). However, no excessive mortality was observed in cultures labeled with SPIO compared to the control after three weeks (Fig. 1). Thus, the data support no adverse effect on cell viability in vitro following incubation and labeling with either, VSOP, Resovist, or Resovist in conjugation with the transfection agent PLL.

In situ labeling. VSOP-labeled tissue in the complete non-amputated limb was clearly visible as hypointense areas after the injection procedure (Fig. 2A, $0 \mathrm{dpa}$ ), as well as in the stump and the emerging blastema following amputation (Fig. 2A, 2-84 dpa). To allow for a quantitative analysis the symmetric Kullback-Leibler divergence was calculated from ROI's originating from the labeled limb stump and blastema relative to the contralateral limb. The logarithmic transformed symmetric Kullback-Leibler divergence approximated a normal distribution. The signal intensity distribution of the SPIO labeled regenerating blastema was significantly different from that of the unlabeled control blastema for the first 48 days of regeneration (Fig. 2B). Initially, the signal intensity distribution of the SPIO labeled limb stump was not significantly different $(\mathrm{P}=0.062)$ from that of the unlabeled control limb, however the signal intensity distribution was significantly different between 2 dpa and 16 dpa (Fig. 2C). From day 28 until the end of the experiment, the signal intensity distribution of the limb stump of labeled and unlabeled animals were no longer significantly different (Fig. 2C).

Histological examinations at the end of the experiment showed remaining iron particles in $33 \%$ of the fully regenerated SPIO labeled limbs all residing proximal to the amputation plane (Fig. 3).

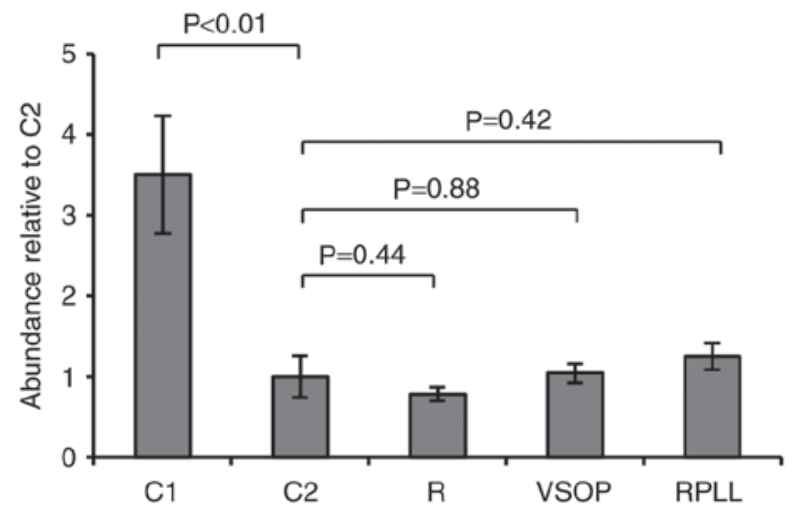

Figure 1. Viability of cultured blastema cells. Pico green determined abundance is set relative to the abundance of control cells after three weeks of culture (C2). A general decline in cell abundance was observed for all groups cultured for three weeks relative to one week (C1), however SPIO labeling with neither Resovist (R), VSOP nor Resovist in combination with a transfection agent (RPLL) affects viability significantly relative to the control group (C2). Values are mean \pm standard error of the mean.

Enhanced permeability and retention. No EPR effects were observed for either Resovist (Fig. 4A) or SPIO-PEG (23 nm) (Fig. 4B) except in the liver of SPIO-PEG $(23 \mathrm{~nm})$ treated animals (Fig. 4B).

Homing of blastema cells. Incubating blastema cells for $24 \mathrm{~h}$ with SPIO-PEG-TF6 $(47 \mathrm{~nm})$ resulted in a clear uptake of iron in the cytoplasm of labeled cells (Fig. 5) with a labeling success of $94 \%$. MRI revealed no significant change in T2 in the blastema of SPIO-PEG-TF6 $(47 \mathrm{~nm})$ treated animals relative to control animals $24 \mathrm{~h}$ after cell injection (Fig. 6A). Instead, a significant decrease in T2 was observed in the liver, heart, and kidney of transplanted animals receiving SPIO-PEG-TF6 $(47 \mathrm{~nm})$ labeled cells (Fig. 6A). Injection of $\mathrm{GFP}^{+}$cells in GFPhosts resulted in detectable $\mathrm{GFP}^{+}$cells only in the kidneys of the GFP- hosts $24 \mathrm{~h}$ post injection (Fig. 6B).

IVIS initially revealed a strong autofluorescent signal from the stomach of both SPIO-PEG-TF6 (47 nm) labeled and unlabeled animals (Fig. 7A and B), possible resulting from the fodder. To avoid premature image saturation this autofluorescent area was covered by black sheets of plastic, however no signal difference was observed between SPIO-PEG-TF6 $(47 \mathrm{~nm})$ treated or control animals from either the blastema or any other underlying organs (Fig. 7C and D). An injection series of increasing concentrations of SPIO-PEG-TF6 $(47 \mathrm{~nm})$ labeled cells was performed subdermally in the tail of previously unlabeled animals, and a concentration as low as 50 cells $/ \mu 1$ allowed for detection of the fluorescent signal (Fig. 7E and G).

Both color Doppler and quadratic averaged B-mode ultrasonographic imaging revealed vascular supply to the proximal part of the early bud blastema (Fig. 8) and a blood flow of $27.2 \pm 5.8 \mu \mathrm{l} / \mathrm{min}$ in the main artery supplying the blastema.

\section{Discussion}

Over the past 20-30 years, stem cell approaches in regenerative medicine have gained ever increasing interest facilitated by the discovery of induced pluripotent stem cells (40). 


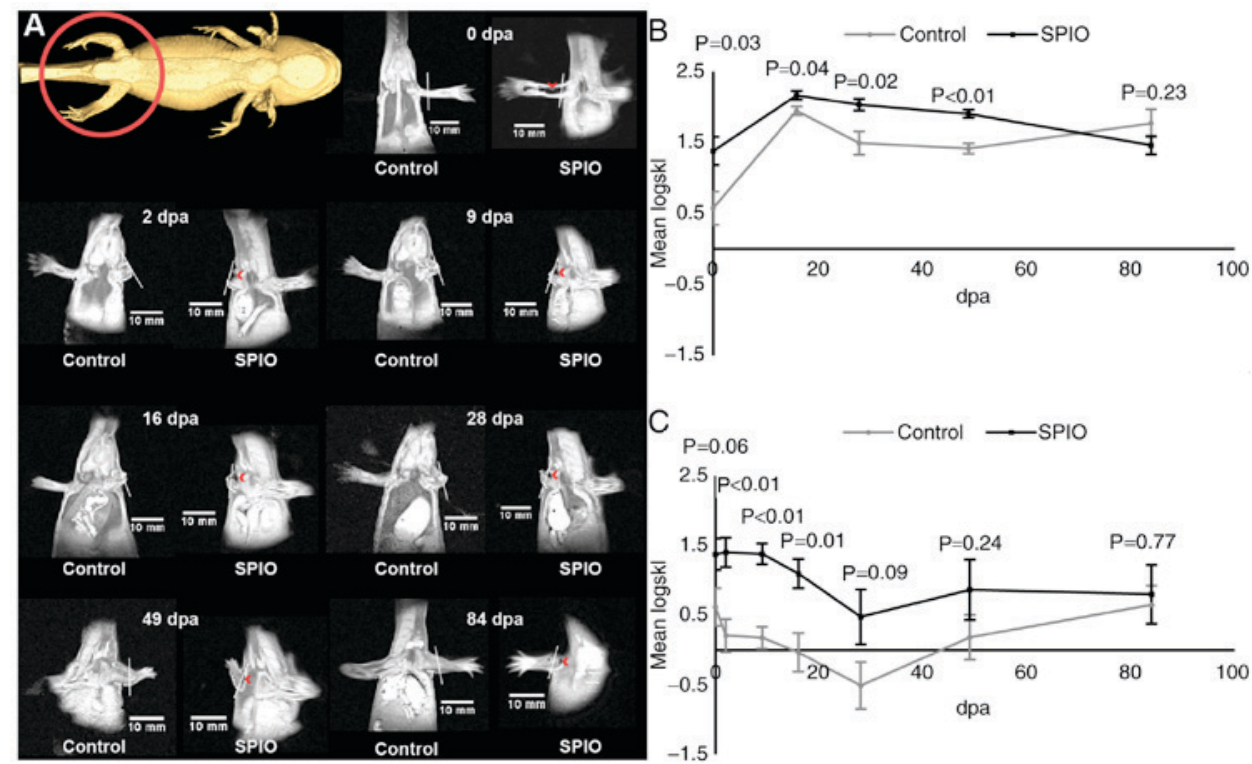

Figure 2. In situ labeling of regenerating axolotl limb at various days post amputation (dpa). Representative MR images at early and late time points (A) and symmetric Kullback-Leibler divergence between in situ labeled (right) and unlabeled (left) blastema (B) and limb stump (C). Initially the signal intensity of the SPIO (VSOP-C200) labeled tissue was significantly different from that of the unlabeled control tissue and appeared as hypointense areas (red arrow heads) in the limb and limb stump (B), however over time the effect of the increasingly diluted SPIO decreased. Values are mean \pm standard error of the mean. White lines in (A) indicate amputation plane. P-values represented Control vs. SPIO.
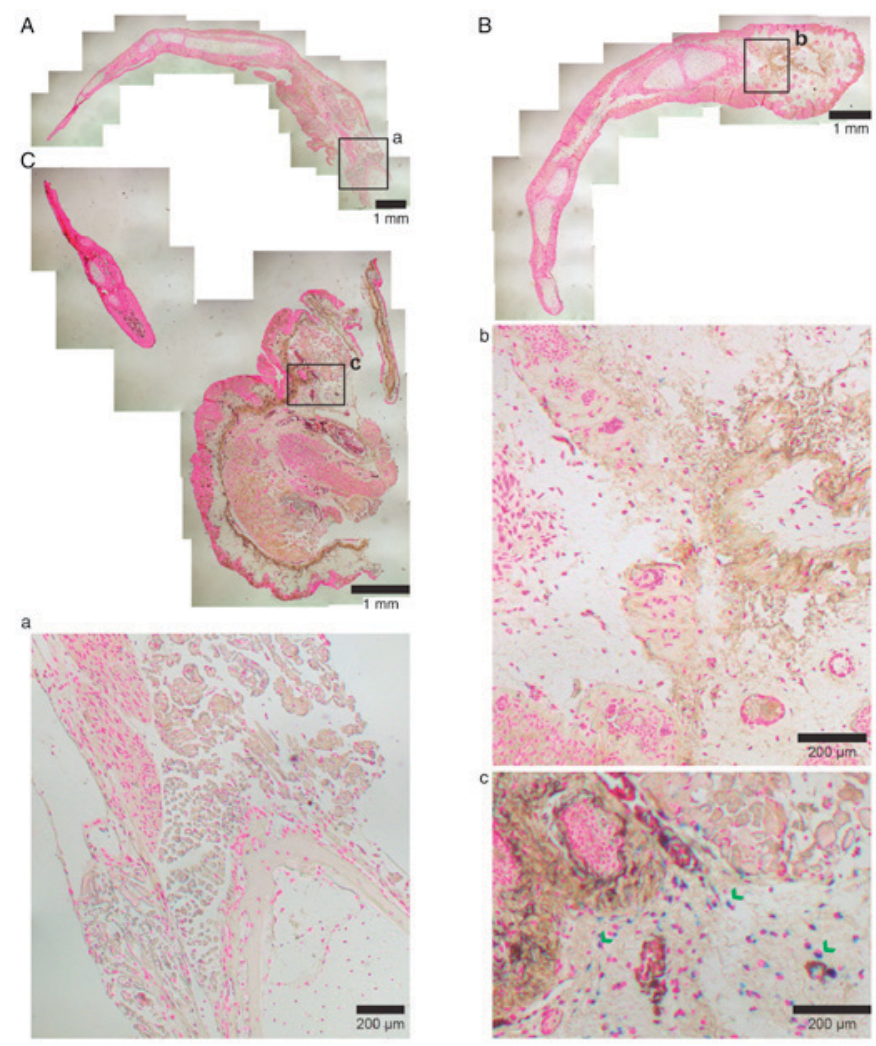

Figure 3. Cellular uptake of SPIOs in tissue. Prussian blue stained longitudinal sections of newly regenerated limbs. (A) Limb of control animal containing no SPIO. (B) Limb of SPIO (VSOP-C200) labeled animal. SPIOs are completely metabolized and are no longer present in this sample. (C) Limb of another SPIO labeled animal. SPIOs are not completely metabolized and iron particles (blue) are still present at the proximal part of the limb (green arrow heads in c).

Nonetheless, with the exception of hematopoietic stem cell transplantations for hematopoietic disorders, most stem cell
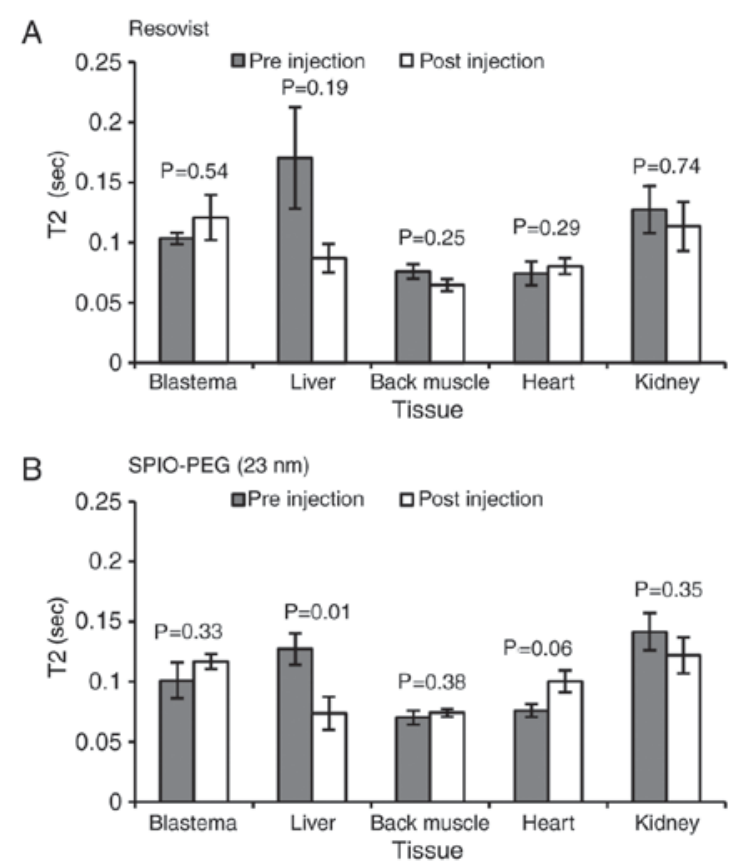

Figure 4. Enhanced permeability and retention effect. The EPR effect of (A) Resovist and (B) SPIO-PEG $(23 \mathrm{~nm})$ on T2 relaxation in the blastema, liver, back muscle, heart, and kidney. EPR only resulted in a significant decrease in T2 in the liver of SPIO-PEG $(23 \mathrm{~nm})$ injected animals. Values are mean \pm standard error of the mean. P-values represented pre injection vs. post inection.

therapies remain experimental and limited therapies are clinically available today (41). A thorough understanding of the route of delivery and subsequent migration of administered stem cells is paramount in the achievement of developing future stem cell therapies in addition to an understanding of cell-cell signaling and requirements for the cellular milieu. This study addresses the use on non-toxic SPIOs to track 
SPIO B

B
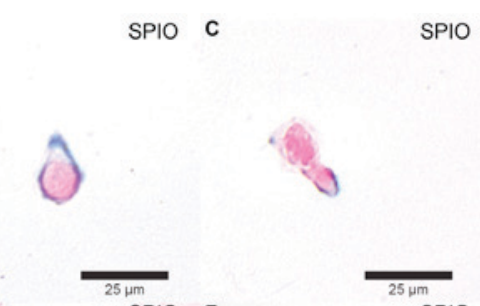

D

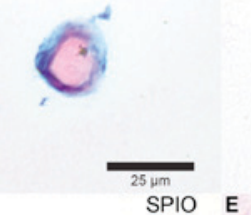

G
SPIO F O

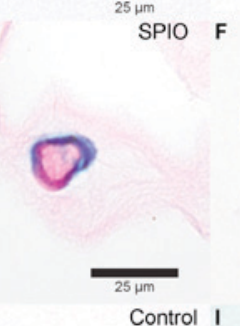

Control ।

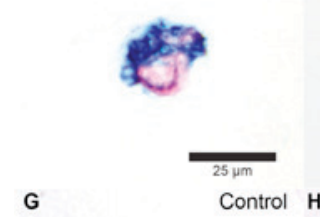

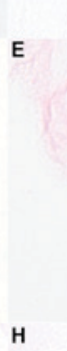

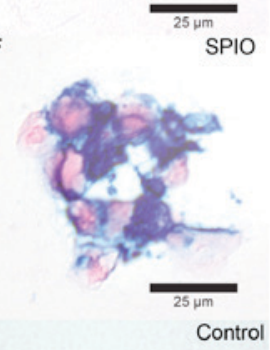

Control
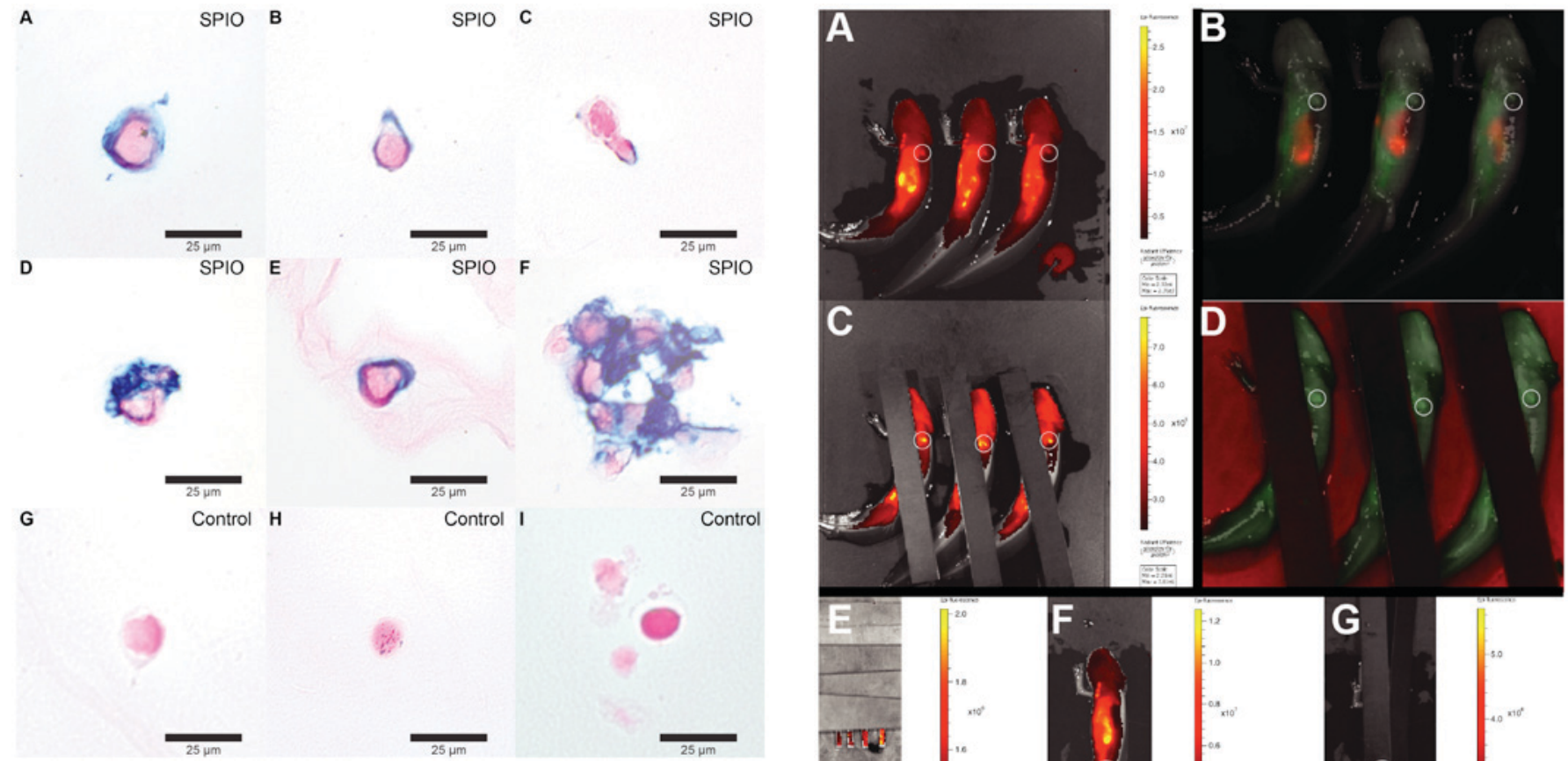

C

D
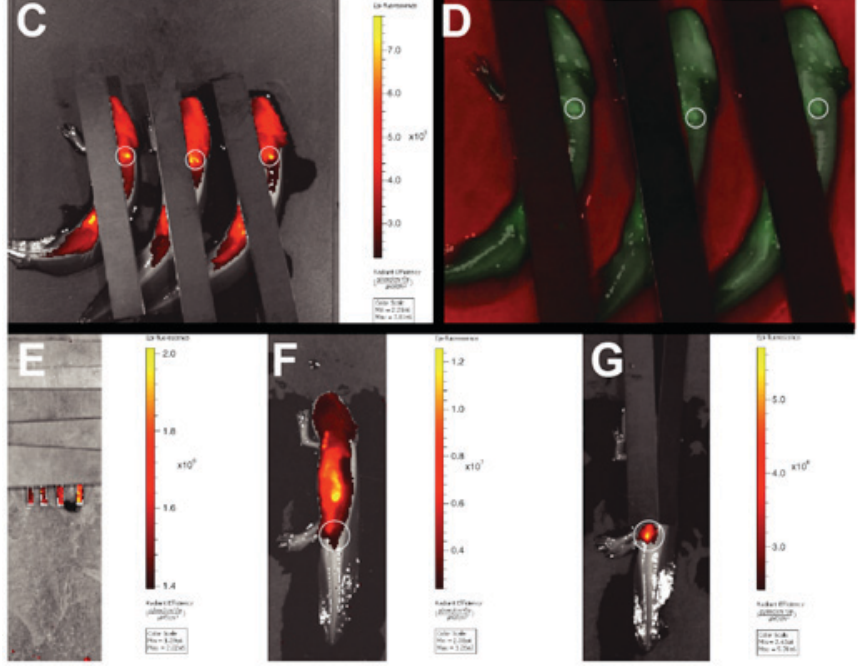

Figure 7. IVIS inspection of animals injected with fluorophore conjugated SPIO (SPIO-PEG-TF6 (47 nm)) labeled blastema cells. Animals in the middle in (A-D) are SPIO treated animals whereas animals to the left and right are control animals injected with unlabeled cells. (A, C, E, F and G) Images captured at the Tide6 optimized emission and excitation wavelengths $(720 / 675 \mathrm{~nm})$. (B and D) Images produced by spectral unmixing employing information from all applied emission and excitation wavelengths. A strong autofluorescent signal was observed from the stomach of both control and SPIO treated animals (A and B), thus it was necessary to cover this part by black sheets of plastic $(\mathrm{C}, \mathrm{D}$ and $\mathrm{G})$ in order not to max out the signal detectors. (E) Increasing concentrations of SPIO-PEG-TF6 (47 nm) labeled cells in capillary tubes (left to right: $0,5,50,500$ cells $/ \mu 1$ ). A concentration of $>50$ cells $/ \mu 1$ yielded a significantly different signal from that of pure water. $20 \mu \mathrm{l}$ of medium containing increasing abundance of SPIO-PEG-TF6 $(47 \mathrm{~nm})$ labeled cells was injected in the tail of previously unlabeled animals. Autofluorescence in the stomach did not allow detection of cells in uncovered animals $(\mathrm{F})$ however covering the stomach with black plastic sheets allowed for detection of cells at a concentration of 50 cells $/ \mu 1(\mathrm{G})$. Circles in (A-D) indicate the position of the blastema; circles in (F) and $(G)$ indicate the injection site of labeled cells.

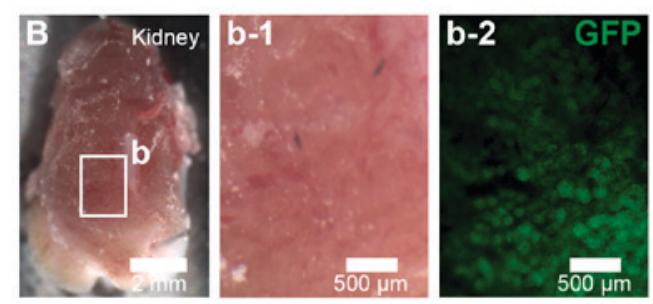

Figure 6. Blastema cell homing and organ filtering. (A) The effect of potentially homing blastema cells on T2 in the blastema, liver, back muscle, heart and kidney. T2 is only significantly shortened in the liver, heart and kidneys as a result of an increased abundance of SPIO (SPIO-PEG-TF6 (47 nm)) labeled cells in these organs. T2 of kidney is represented on a secondary second axis due to larger values. Values are mean \pm standard error of the mean. (B) Injection of $\mathrm{GFP}^{+}$blastema cells in $\mathrm{GFP}^{-}$animals lead to a highly detectable capture of $\mathrm{GFP}^{+}$blastema cells in the kidneys. P-values represented Control vs. SPIO-PEG-TF6 (47 nm).

administered cells non-invasively in an intrinsic regenerative environment, the regenerating limb of the axolotl. Overall, we found that SPIOs are applicable for cell tracking in this animal model, and this was used to demonstrate the lack of an early homing mechanism of blastema cells to the zone of regeneration.

Dissociated axolotl blastema cells do not tolerate extended periods of culturing outside their natural environment well (18). Therefore, a general decline in cell numbers of 3 weeks old cultures relative to 1 week old cultures is anticipated. However, culturing blastema cells with either of two commercially available SPIOs and the transfection agent PLL did not affect viability (Fig. 1). Also, no malformations or decrease in regenerative rate were observed throughout the experiments involving SPIOs. Concerns have been raised that loading cells with huge amounts of even non-toxic metals such as iron can affect gene expression (34). We did not test such effects in the present study, and cannot rule out transcriptomic effects of SPIOs in axolotl blastema cells, although we 


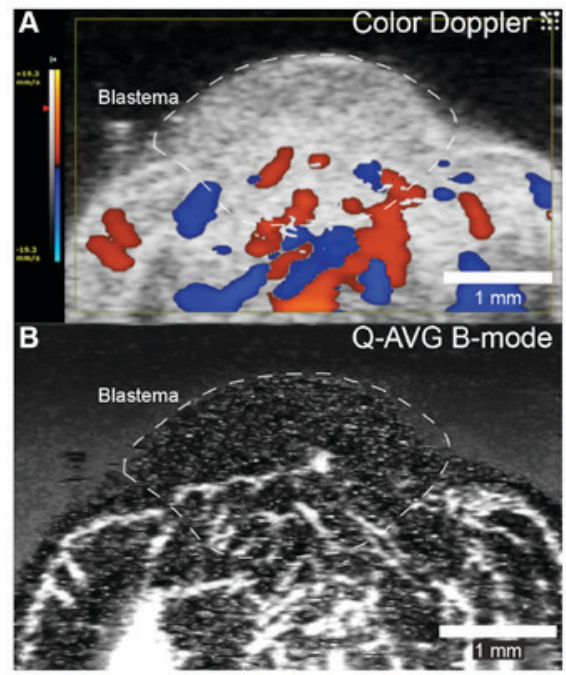

Figure 8. Vasculature in early bud blastema. (A) Color Doppler ultrasound image and (B) quadratic averaged B-mode image of a representative early bud blastema (distal towards the top). The blastema is already highly vascularized. Red indicates blood running towards the transducer in the color Doppler image, blue in the opposite direction.

observed no effects on viability and phenotypic regeneration to imply this and it is noteworthy that the majority of studies applying SPIOs for cell labeling report of no or very little toxicity of these particles (24-26).

To test the feasibility and longevity of SPIO labeling in the axolotl, we initially performed in situ labeling, by injecting a small amount of highly concentrated particles in the limb, followed by amputation, and subsequently monitored the regenerative process using MRI. The applied SPIOs proved effective for labeling cells in situ for the initial phases of regeneration (Fig. 2). Kullback-Leibler analysis was able to detect significant differences in signal distribution of the regenerating blastema all the way through the regeneration of the miniature limb until 48 days after amputation, and the presence of SPIO labeled cells proximal to the amputation zone was detectable with MRI until 16 days after amputation. A decrease in signal of SPIO labeled proliferating cells is to be expected due the dilution of iron in each cell as the cytoplasm is divided between new daughter cells (42). In the regrowth period between day 48 and day 84 post amputation all limb elements are already in place, but the high degree of hyperplasia needed to restore the normal sized limb may explain the dilution of SPIO to a level below the detection limit in the blastema in this period. Iron nanoparticles have been described to ultimately become metabolized in the body $(30,42,43)$ and thus we anticipated a general decrease of concentration i.e., MRI signal over time, especially in tissues were macrophages congregate such as in the limb stump after amputation (44).

Limb regeneration in the salamander relies on the proliferative capacity of differentiated cells from various lineages residing local to the site of amputation rather than a source of circulating stem cells that find their way to injury sites (13). It has been suggested that a positional coding of primarily fibroblast enables the regenerative environment to restore the exact anatomy of the injured extremity, however the complete mechanisms are still not fully understood (20). We were interested in the capacity of blastema cells to recognize a site of injury and hypothesized that blastema cells injected in the blood pool would relatively rapidly congregate at an injury site given that an adequate supply of blood vessels was restored. This hypothesis was addressed by injecting a large quantity of SPIO labeled blastema cells at early bud stage. At this time a capillary network is established supporting the budding limb (Fig. 8), and based on the flow measurements in the main artery supplying the blastema, the total blood mass (an estimated 5\% of body mass) passes through these vessels in every $1.5 \mathrm{~h}$. Nevertheless, we were not able to detect any signal change as a result of an up-concentration of fluorophore conjugated SPIOs with neither of our two in vivo techniques (MRI and IVIS). It is worth noting that potentially homing blastema cells would only need to penetrate through the capillary endothelium at an increased rate in the regeneration zone for these two techniques to detect an accumulation here, the cells would not need to penetrate through avascular tissue in the distal part of the blastema which may take longer than $24 \mathrm{~h}$. Instead, labeled and injected blastema cells seem to become retained in organs normally responsible for metabolizing administered agents as part of the detoxification processes such as the liver and the kidneys, the latter being most prevailing in this study. This was further supported by injecting GFP expressing blastema cells in non-GFP hosts. Although this was a somewhat crude method based on the fluorescence of entire organs that possibly only allows for the detection of a large number of florescent cells, the signal from the kidneys was in fact so strong that it could be picked up (Fig. 6B) supporting the result of the MRI experiment. Injected cells were also found to be caught in the heart (Fig. 6A), which seems likely due to the spongious nature of the amphibian heart and may also be due to the cardiac injection of SPIO labelled cells. While our data suggest that there is no short term homing effect of circulating blastema cells over the course of $24 \mathrm{~h}$ (16 cycles of total blood volume through the regenerating limb), we cannot rule out potential long term homing effects and if blastema cells that are at first caught in the kidneys, liver and heart later migrate to the budding limb.

Concerns have been raised that SPIOs remain to function as contrast agents for MRI even if initially labeled cells are dead and the SPIOs are ingested in other cells or concentrate passively via the EPR effect in organs (45). We tested the potential EPR effect of two of the SPIOs used in this study [Resovist and SPIO-PEG $(23 \mathrm{~nm})]$ and found an up-concentration of one of the SPIOs [SPIO-PEG (23 nm)] in the liver. A possible explanation for this observation is that SPIOs are known to be metabolized in the liver (30), and therefore a short term response is seen in this organ when injected iron is rapidly accumulated and cleared. Additionally, we observed a decrease in T2 (i.e., an increased iron concentration) in the liver, kidneys and the spongious heart (the latter may be due to the cardiac injection of the particles) after injection of SPIO labeled blastema cells. Therefore, we expect the escape rate of SPIOs out of labeled blastema cells to be insignificant, and the up-concentration of SPIOs in filter organs to be a result of a passive increase in the number of labeled cells in these organs and not free SPIO particles.

Another concern regarding the use of SPIO particles in cell tracking experiments is the fact that the iron-oxide based core acts as a negative contrast agent, and therefore one is looking for a decrease in signal at the presence of SPIO particles in a structure rather than an increase which is the case with e.g., 
gadolinium based contrast agents for MRI. This raises the concern that the presence of SPIO particles at a specific point of interest can be mistaken by other sources of susceptibility artifacts such as blood clots, hemosiderin complexes, small gas bubbles etc. Therefore, a rigid control setup, as applied in this study, in which control animals undergo the exact same surgical and experimental procedures as the animals receiving SPIO particles, is essential to rule out the possibility of falsely interpreting other sources of susceptibility artefacts as the presence of SPIOs at a given location.

It the present study we have applied SPIO labeling to address the biological question whether stem cell like blastema cells in a regeneration competent animal are able to congregate at a regeneration site (the blastema) when applied intravascularly after $24 \mathrm{~h}$ of circulation in the vascular system. We found no indications of this on the short term, instead injected cells were captured in the kidneys, liver and the spongious heart. There is a considerable evolutionary distance between salamanders and mammals, and from this study it is not possible to occlude vascular injections as a viable way for stem cell delivery in regenerative therapies. However, we believe that it should be taking into account when planning future research efforts involving the injection of circulating stem cells that this is seemingly not the important mechanism in regeneration competent organisms such as the axolotl, or at least we have not been able to observe this over the course of our experiment, therefore it may be more fruitful to put emphasis on therapies acting on the local damaged environment.

In the present study we tested the applicability of SPIO-labeling to track stem-like cells non-invasively in an intrinsic regenerative environment, the limb of the axolotl. SPIOs showed no effect on viability and function of labeled cells and proved useful for non-invasively tracking the fate of vascular injected cells. SPIOs were applied to investigate whether blastema cells have the potential to act as circulating stem cells homing at sites of injury. We observed no accumulation of labeled blastema cells in the zone of regeneration which indicates that this mechanism is not at play in the restoration of a limb in the axolotl or that the accumulation of blastema cells at the regenerative zone is a very slow process that falls out of the time regime of this experiment.

\section{References}

1. Birnbaum KD and Sánchez-Alvarado A: Slicing across kingdoms: Regeneration in plants and animals. Cell 132: 697-710, 2008.

2. Edwards RG: From embryonic stem cells to blastema and MRL mice. Reprod Biomed Online 16: 425-461, 2008.

3. Stocum DL: Regenerative biology and medicine. J Musculoskelet Neuronal Interact 2: 270-273, 2002.

4. Stocum DL and Zupanc GK: Stretching the limits: Stem cells in regeneration science. Dev Dyn 237: 3648-3671, 2008.

5. Douglas BS: Conservative management of guillotine amputation of the finger in children. Aust Paediatr J 8: 86-89, 1972.

6. Goss RJ, Van Praagh A and Brewer P: The mechanism of antler casting in the fallow deer. J Exp Zool 264: 429-436, 1992.

7. Goss RJ: Future directions in antler research. Anat Rec 241: 291-302, 1995.

8. Han M, Yang X, Lee J, Allan CH and Muneoka K: Development and regeneration of the neonatal digit tip in mice. Dev Biol 315: 125-135, 2008.

9. Huh JY, Choi BH, Kim BY, Lee SH, Zhu SJ and Jung JH: Critical size defect in the canine mandible. Oral Surg Oral Med Oral Pathol Oral Radiol Endod 100: 296-301, 2005.
10. Rosenthal LJ, Reiner MA and Bleicher MA: Nonoperative management of distal fingertip amputations in children. Pediatrics 64: 1-3, 1979.

11. Krogh A: The progress of physiology. Science 70: 200-204, 1929.

12. Bryant SV, Endo T and Gardiner DM: Vertebrate limb regeneration and the origin of limb stem cells. Int J Dev Biol 46: 887-896, 2002.

13. Kragl M, Knapp D, Nacu E, Khattak S, Maden M, Epperlein HH and Tanaka EM: Cells keep a memory of their tissue origin during axolotl limb regeneration. Nature 460: 60-65, 2009.

14. Menger B, Vogt PM, Kuhbier JW and Reimers K: Applying amphibian limb regeneration to human wound healing: A review. Ann Plast Surg 65: 504-510, 2010.

15. Morrison JL, Lööf S, He P and Simon A: Salamander limb regeneration involves the activation of a multipotent skeletal muscle satellite cell population. J Cell Biol 172: 433-440, 2006.

16. Roy S and Gatien S: Regeneration in axolotls: A model to aim for! Exp Gerontol 43: 968-973, 2008.

17. Stoick-Cooper CL, Moon RT and Weidinger G: Advances in signaling in vertebrate regeneration as a prelude to regenerative medicine. Genes Dev 21: 1292-1315, 2007.

18. Armstrong JB and Malacinski GM: Developmental Biology of the Axolotl. Barnes Noble 65: 336, 1989.

19. Gresens J: An introduction to the Mexican axolotl (Ambystoma mexicanum). Lab Anim (NY) 33: 41-47, 2004.

20. Endo T, Bryant SV and Gardiner DM: A stepwise model system for limb regeneration. Dev Biol 270: 135-145, 2004.

21. Kumar A, Nevill G, Brockes JP and Forge A: A comparative study of gland cells implicated in the nerve dependence of salamander limb regeneration. J Anat 217: 16-25, 2010.

22. Satoh A, James MA and Gardiner DM: The role of nerve signaling in limb genesis and agenesis during axolotl limb regeneration. J Bone Joint Surg Am 91 (Suppl 4): S90-S98, 2009.

23. Tank PW, Carlson BM and Conelly TG: A staging system for forelimb regeneration in the axolotl, Ambystoma mexicanum. J Morphol 150: 117-128, 1976.

24. Arbab AS, Jordan EK, Wilson LB, Yocum GT, Lewis BK and Frank JA: In vivo trafficking and targeted delivery of magnetically labelled stem cells. Hum Gene Ther 15: 351-360, 2004.

25. Arbab AS, Yocum GT, Kalish H, Jordan EK, Anderson SA, Khakoo AY, Read EJ and Frank JA: Efficient magnetic cell labelling with protamine sulfate complexed to ferumoxides for cellular MRI. Blood 104: 1217-1223, 2004.

26. Saldanha KJ, Piper SL, Ainslie KM, Kim HT and Majumdar S: Magnetic resonance imaging of iron oxide labelled stem cells: Applications to tissue engineering based regeneration of the intervertebral disc. Eur Cell Mater 16: 17-25, 2008.

27. Crevensten G, Walsh AJ, Ananthakrishnan D, Page P, Wahba GM, Lotz JC and Berven S: Intervertebral disc cell therapy for regeneration: Mesenchymal stem cell implantation in rat intervertebral discs. Ann Biomed Eng 32: 430-434, 2004.

28. Sakai D, Mochida J, Iwashina T, Hiyama A, Omi H, Imai M, Nakai T, Ando K and Hotta T: Regenerative effects of transplanting mesenchymal stem cells embedded in atelocollagen to the degenerated intervertebral disc. Biomaterials 27: 335-345, 2006.

29. Haacke EM, Brown RW, Thompson MR and Vankatesan R: Magnetic Resonance Imaging. Med diag, 1999.

30. Ittrich H, Peldschus K, Raabe N, Kaul M and Adam G: Superparamagnetic iron oxide nanoparticles in biomedicine: Applications and development in diagnostics and therapy. Rofo 185: 1149-1166, 2013.

31. Frank JA, Miller BR, Arbab AS, Zywicke HA, Jordan EK, Lewis BK, Bryant LH Jr and Bulte JW: Clinically applicable labelling of mammalian and stem cells by combining superparamagnetic iron oxides and transfection agents. Radiology 228: 480-487, 2003.

32. Kalish H, Arbab AS, Miller BR, Lewis BK, Zywicke HA, Bulte JW, Bryant LH Jr and Frank JA: Combination of transfection agents and magnetic resonance contrast agents for cellular imaging: Relationship between relaxivities, electrostatic forces, and chemical composition. Magn Reson Med 50: 275-282, 2003.

33. Yang CY, Tai MF, Lin CP, Lu CW, Wang JL, Hsiao JK and Liu HM: Mechanism of cellular uptake and impact of ferucarbotran on macrophage physiology. PLoS One 6: e25524, 2011.

34. Foldager CB, Pedersen M, Ringgaard S, Bünger C and Lind M: Chondrocyte gene expression is affected by very small iron oxide particles-labeling in long-term in vitro MRI tracking. J Magn Reson Imaging 33: 724-730, 2011. 
35. Kafuels N, Korn R, Wagner S, Schink T, Hamm B, Taupitz M and Schnorr J: Magnetic resonance imaging of liver metastases: Experimental comparison of anionic and conventional superparamagnetic iron oxide particles with a hepatobiliary contrast medium during dynamic and uptake phases. Invest Radiol 43: 496-503, 2008.

36. Hansen L, Larsen EK, Nielsen EH, Ivesen F, Liu Z, Thomsen K, Pedersen M, Skrydstrup T, Nielsen NC, Ploug M and Kjems J: Targeting of peptide conjugated magnetic nanoparticles to urokinase plasminogen activator receptor (uPAR) expressing cells. Nanoscale 5: 8192-8201, 2013.

37. Kumar A and Godwin JW: Preparation and culture of limb blastema stem cells from regenerating larval and adult salamanders. Cold Spring Harb Protoc 2010: pdb. prot5367, 2010.

38. Cosden RS, Lattermann C, Romine S, Gao J, Voss SR and MacLeod JN: Intrinsic repair of full-thickness articular cartilage defects in the axolotl salamander. Osteoarthritis Cartilage 19: 200-205, 2011

39. Kullback $S$ and Leibler RA: On information and sufficiency. Ann Math Stat 22: 79-86, 1951.

40. Takahashi K and Yamanaka S: Induction of pluripotent stem cells from mouse embryonic and adult fibroblast cultures by defined factors. Cell 126: 663-676, 2006.
41. Ratcliffe E, Glen KE, Naing MW and Williams DJ: Current status and perspectives on stem cell-based therapies undergoing clinical trials for regenerative medicine: Case studies. Br Med Bull 108: 73-94, 2013.

42. Qi Y, Feng G, Huang Z and Yan W: The application of super paramagnetic iron oxide-labeled mesenchymal stem cells in cell-based therapy. Mol Biol Rep 40: 2733-2740, 2013.

43. Weissleder R, Stark DD, Engelstad BL, Bacon BR, Compton CC, White DL, Jacobs P and Lewis J: Superparamagnetic iron oxide: Pharmacokinetics and toxicity. AJR Am J Roentgenol 152: 167-173, 1989.

44. Godwin JW, Pinto AR and Rosenthal NA: Macrophages are required for adult salamander limb regeneration. Proc Natl Acad Sci USA 110: 9415-9420, 2013.

45. Winter EM, Hogers B, van der Graaf LM, Gittenberger-de Groot AC, Poelmann RE and van der Weerd L: Cell tracking using iron oxide fails to distinguish dead from living transplanted cells in the infarcted heart. Magn Reson Med 63: 817-821, 2010.

This work is licensed under a Creative Commons

Attribution-NonCommercial-NoDerivatives 4.0 International (CC BY-NC-ND 4.0) License. 